\title{
Adults with early-onset type 2 diabetes (aged 18-39 years) are severely underrepresented in diabetes clinical research trials
}

\author{
Jack A. Sargeant ${ }^{1,2}$ (D) Emer M. Brady ${ }^{1,3}$ (D) - Francesco Zaccardi ${ }^{1,4} \cdot$ Frances Tippins $^{1} \cdot$ David R. Webb $^{1,2}$. \\ Vanita R. Aroda ${ }^{5}$. Edward W. Gregg ${ }^{6}$ - Kamlesh Khunti ${ }^{1,7} \cdot$ Melanie J. Davies ${ }^{1,2}$ (D)
}

Received: 10 December 2019 / Accepted: 6 April 2020/Published online: 2 June 2020

(C) The Author(s) 2020

\begin{abstract}
Aims/hypothesis Early-onset adult type 2 diabetes (diagnosed between ages 18 and 39 years) is increasingly prevalent and associated with poor long-term outcomes. We hypothesised that individuals with early-onset adult type 2 diabetes were underrepresented in the prominent research trials that underpin type 2 diabetes management guidelines.

Methods We reviewed the mean age of the study populations recruited to 90 prominent trials in type 2 diabetes, including 37 cardio-renal outcomes trials across a range of pharmacological, non-pharmacological and multifactorial interventions, 28 trials from the phase III programmes of three representative glucose-lowering therapies used routinely in clinical practice (empagliflozin, liraglutide and sitagliptin) and 25 prominent trials of diabetes self-management education and support or intensive lifestyle interventions (diet or supervised exercise training). We then estimated the number of individuals within these trials who were aged between 18 and 39 years.

Results Across all 90 trials, the mean age of 268,978 participants was 63 years (range 51-69 years in individual trials). In 73 trials $(81 \%),<5 \%$ of participants were estimated to be aged 18-39 years, despite this age group representing $\sim 15-20 \%$ of the adult type 2 diabetes population. Twenty-nine of these trials (32\%; total 164,953 participants) excluded individuals below 40 years of age altogether. Conclusions/interpretation Guidelines for early-onset adult type 2 diabetes are extrapolated predominantly from evidence in older individuals. Strategies to support the participation of individuals with early-onset adult type 2 diabetes in future research are imperative to ensure guidelines for these high-risk individuals are evidence-based.
\end{abstract}

Keywords Clinical research $\cdot$ Clinical trials $\cdot$ Early-onset type 2 diabetes $\cdot$ Representation

Electronic supplementary material The online version of this article (https://doi.org/10.1007/s00125-020-05174-9) contains peer-reviewed but unedited supplementary material, which is available to authorised users.

Jack A. Sargeant js928@leicester.ac.uk

1 Diabetes Research Centre, University of Leicester, Leicester General Hospital, Gwendolen Road, Leicester LE5 4PW, UK

2 NIHR Leicester Biomedical Research Centre, University Hospitals of Leicester NHS Trust and the University of Leicester, Leicester, UK

3 Department of Cardiovascular Sciences, University of Leicester, Leicester, UK

4 Real-World Evidence Unit, University of Leicester, Leicester, UK

5 Brigham and Women's Hospital, Harvard Medical School, Boston, MA, USA

6 Department of Epidemiology and Biostatistics, School of Public Health, Imperial College London, London, UK

7 NIHR Applied Research Collaboration East Midlands, Leicester, UK

\section{Abbreviations}

DSMES Diabetes self-management education and support GLT Glucose-lowering therapy

\section{Introduction}

The prevalence of type 2 diabetes, traditionally considered a condition of mid-to-late adulthood, is increasing in younger adults (aged 18-39 years, inclusive) [1]. It is estimated that these individuals with 'early-onset adult type 2 diabetes' now represent up to $15-20 \%$ of the adult type 2 diabetes population worldwide [1-3]. Early-onset adult type 2 diabetes is underpinned by an extreme risk phenotype, early exposure to chronic hyperglycaemia and suboptimal self-care practices [4-6]; its impact is severe, leading to devastating micro- and macrovascular complications [1, 5, 7, 8]. Psychosocial complications including anxiety, depression and diabetes- 


\section{Research in context}

\section{What is already known about this subject?}

- The number of people diagnosed with type 2 diabetes earlier in life is increasing, including adults aged 18-39 years who now represent $\sim 15-20 \%$ of the adult type 2 diabetes population

- Individuals with 'early-onset adult type 2 diabetes' are a particularly high-risk group, with high levels of multimorbidity and poor long-term outcomes

- However, the number of adults with early-onset type 2 diabetes taking part in clinical research may be limited

What is the key question?

- Are adults with early-onset type 2 diabetes fairly represented in clinical trials underpinning management guidelines for adult type 2 diabetes?

What are the new findings?

- In 90 clinical trials, including those examining pharmacological, diabetes self-management, lifestyle (diet or exercise) or multifactorial interventions, the mean age of 268,978 participants was 63 years

- In $81 \%$ of these trials, $<5 \%$ of the study population was estimated to be aged $18-39$ years (maximum $13 \%$ )

How might this impact on clinical practice in the foreseeable future?

- Management guidelines for early-onset adult type 2 diabetes must be extrapolated from evidence in older individuals; strategies supporting adults with early-onset type 2 diabetes to participate in clinical research are essential to inform optimal diabetes management for this high-risk group

related distress are also highly prevalent $[9,10]$. Moreover, whilst mortality rates in type 2 diabetes are generally in decline, those in early-onset adult type 2 diabetes may be being left behind [11].

$\mathrm{Ke}$ and colleagues [12] recently highlighted that the mean age at type 2 diabetes diagnosis of individuals enrolled in 19 cardiovascular or renal ('cardio-renal') outcomes trials of glucose-lowering therapies (GLTs) was approximately 5060 years. This is concerning because trials such as these heavily inform management guidelines and these guidelines are not age specific [13]. Consequently, guidelines for the use of these therapies in early-onset adult type 2 diabetes must be extrapolated from evidence in older individuals. We hypothesised that this underrepresentation of individuals with early-onset adult type 2 diabetes was not unique to these 19 trials but extended to the wider evidence base underpinning management guidelines.

\section{Methods}

This short communication presents analyses that both complement and extend those presented by $\mathrm{Ke}$ and colleagues [12]. First, we reviewed the baseline age (i.e. age at enrolment) of participants in 37 cardio-renal outcomes trials in type 2 diabetes, across a range of pharmacological, non-pharmacological and multifactorial interventions. Our focus on age at enrolment represents a subtle but important, complementary difference from the analyses of $\mathrm{Ke}$ and colleagues, as individuals aged 18-39 years often have distinct sociocultural circumstances that may contribute to their lower representation in clinical research (e.g. family planning, early careers, new independent living). Analyses of age at diagnosis (including those by Ke and colleagues) provide valuable insight into the representation of early-onset adult type 2 diabetes as a disease phenotype within trial populations (i.e. inclusion of people diagnosed between the ages of 18 and 39 years), but nonetheless many of these participants may be older at the point of enrolment (e.g. a 50-year-old individual with disease duration of 15 years).

Second, our review extends to studies beyond just cardiorenal outcomes trials, by incorporating a representative sample of trials that underpin other fundamental components of adult type 2 diabetes management [13], including: (1) trials within the phase III research programmes of three GLTs used routinely in clinical practice and which are representative of modern phase III research programmes (the sodium-glucose co-transporter 2 inhibitor empagliflozin, the glucagon-like peptide-1 receptor agonist liraglutide, and the dipeptidyl peptidase-4 inhibitor sitagliptin); (2) prominent trials examining the efficacy of diabetes self-management education and support (DSMES) and intensive lifestyle interventions (diet or supervised exercise) in adults with type 2 diabetes.

Third, where possible, we complement our review of baseline age by estimating the number of individuals aged 1839 years within the trials included. In manuscripts that 
reported the age of the recruited population as a mean (thus allowing the assumption of a normal distribution of data), we used this mean, the SD and an online calculator (http:// onlinestatbook.com/2/calculators/normal_dist.html) to estimate the proportion of individuals aged 18-39 years enrolled in each trial. We then calculated this proportion of the total study sample size to estimate the number of individuals aged 18-39 years recruited.

Further details of the search strategy, study selection and data extraction are presented in electronic supplementary material (ESM) Methods.

\section{Results}

Thirty-seven cardio-renal outcomes trials were identified, examining the effects of intensive glucose lowering towards pre-specified targets $(n=5)$, specific pharmacological GLTs $(n=27)$, and lifestyle $(n=1)$, surgical $(n=1)$ or multifactorial interventions $(n=3)$. Twenty-eight phase III trials of GLTs (empagliflozin $n=9$, liraglutide $n=7$, sitagliptin $n=12$ ) were also included, along with 25 prominent trials of DSMES $(n=$ 13) or intensive lifestyle interventions (diet $n=6$, supervised exercise $n=6$ ). Details of each trial, along with full citations, are provided in ESM Table 1. Collectively, these 90 trials recruited 268,978 individuals, with the mean age of each study population ranging from 51 to 69 years. The mean age of all trials combined, weighted for differences in sample size, was 63 years.

Twenty-three cardio-renal outcomes trials $(62 \%$; total 161,608 participants) had minimum age criteria $\geq 40$ years, thus preventing the inclusion of individuals aged 18 39 years altogether. Nine trials had minimum age criteria of $25(n=3), 30(n=5)$ or $35(n=1)$ years, allowing the inclusion of some, but not all, individuals aged 18-39 years. Only five trials (14\%) allowed the inclusion of all adults $\geq 18$ years.

In the 14 trials for which at least some individuals aged 18 39 years were eligible (i.e. minimum age criteria $<40$ years; total 76,650 participants), the mean age of the recruited populations ranged from 53 to 66 years, with a weighted mean age of 62 years. Twelve of these 14 trials reported age as the mean and $\mathrm{SD}$, thus allowing estimation of the number of individuals aged 18-39 years (the remaining two trials reported the median with or without interquartile range) (ESM Table 1, refs 32 and 39). Collectively, $<1 \%$ of the combined population in these 12 trials were estimated to fall within the ages of 18 39 years (range $0.2-4.8 \%$ ) (Fig. 1). Data for each trial are presented in ESM Fig. 1.

All 28 phase III trials of GLTs (total 18,974 participants) had minimum age criteria of 18 years, except one which was 21 years. The mean age of recruited participants ranged from 51 to 68 years, with a weighted mean age of 56 years. Data stratified by GLT are provided in ESM Table 2. Results
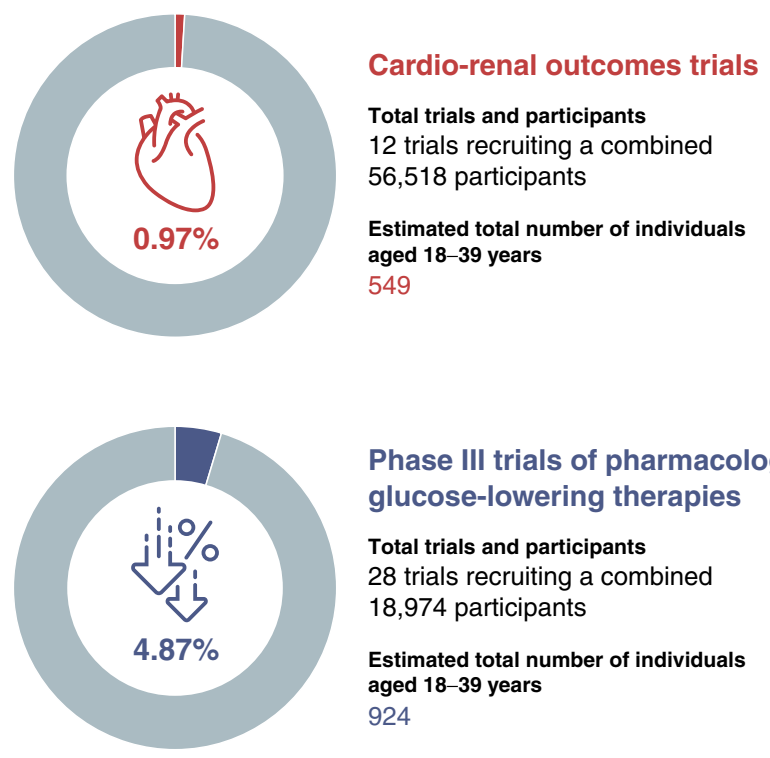

Phase III trials of pharmacological glucose-lowering therapies

Total trials and participants 28 trials recruiting a combined 18,974 participants

Estimated total number of individuals aged 18-39 years 924

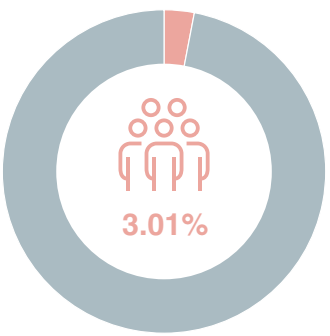

Large trials of DSMES or intensive lifestyle interventions

Total trials and participants 17 trials recruiting a combined 7565 participants

Estimated total number of individuals aged $18-39$ years

Fig. 1 Estimated proportions of individuals aged 18-39 years participating in cardio-renal outcomes trials, phase III trials of pharmacological GLTs used routinely in clinical practice, and prominent trials exploring the efficacy of DSMES or intensive lifestyle interventions (diet or supervised exercise training) in adults with type 2 diabetes. Analyses in cardiorenal outcomes and DSMES or intensive lifestyle intervention trials include only trials for which at least some individuals aged 18-39 years would have been eligible (i.e. minimum age criteria $<40$ years). A further 23 cardio-renal outcomes trials (total 161,608 participants) and six DSMES/intensive lifestyle intervention trials (total 3345 participants) had minimum age criteria $\geq 40$ years, thus excluding individuals aged 18-39 years altogether. Two cardio-renal outcomes trials (total 20,132 participants) and two DSMES trials (total 836 participants) were also excluded from analyses as they did not report age as mean and SD, thus preventing estimation of the proportion of individuals aged 18-39 years

remained similar after exclusion of three trials that specifically recruited individuals with additional comorbidities (hypertension $n=1$, renal insufficiency $n=2$ ), which had notably higher mean ages (ESM Table 1, refs 61, 62 and 81). It was estimated that individuals aged $18-39$ years represented $<5 \%$ of the combined population (range $0.2-13.0 \%$ ) (Fig. 1; ESM Fig. 2).

Six DSMES or intensive lifestyle intervention trials (24\%; total 3345 participants) excluded individuals aged 18 39 years. The minimum age criteria of the remaining trials were $18(n=6), 20(n=1), 21(n=1), 25(n=1), 30(n=5)$, $35(n=1)$ and $39(n=1)$ years. Three trials did not report 
minimum age criteria, but inferred that all individuals with type 2 diabetes were eligible. In the 19 studies with minimum age criteria $<40$ years (total 8401 participants), the weighted mean age was 59 years (range 52-69 years). Stratified data for DSMES, dietary and supervised exercise interventions are provided in ESM Table 3.

Two studies did not report the SD, preventing estimation of the number of individuals aged 18-39 years. It was estimated that approximately $3 \%$ of the combined population in the remaining 17 trials were aged 18-39 years (range 0.0111.2\%) (Fig. 1; ESM Fig. 3).

\section{Discussion}

Our analyses highlight that individuals with early-onset adult type 2 diabetes, aged 18-39 years, are severely underrepresented in clinical research trials. Importantly, this is not isolated to cardio-renal outcomes trials of pharmacological GLTs but extends to similar outcomes trials examining nonpharmacological and multifactorial approaches, as well as phase III trials of GLTs used routinely in clinical practice and other large trials of DSMES or intensive lifestyle interventions. Notably, our analyses include individuals who remained within the ages of 18-39 years at trial enrolment and exclude individuals who were diagnosed with earlyonset adult type 2 diabetes but were $\geq 40$ years when enrolled.

Our findings are important as they suggest that the older age of populations seen in cardio-renal outcomes trials may not solely be a result of purposive eligibility criteria designed to increase statistical power by observing higher rates of adverse cardio-renal events. Other factors, including the often complex busy lives of individuals aged 18-39 years and with potentially differing priorities from those of older adults, may preclude their involvement in clinical research even when eligibility criteria permit their enrolment. Some of these factors (including independent living, lower perceived vulnerability and less established future plans) may be similar to those identified in adolescents with type 2 diabetes (defined as age 15-19 years) [14]. Given that women aged 18-39 years are of childbearing age, family planning also remains an important factor that may preclude their involvement. It is imperative that strategies are implemented to specifically support the effective participation of individuals aged 1839 years in clinical diabetes research trials, where safe, to ensure that management guidelines are appropriately evidence-based, particularly given that the number of individuals with early-onset adult type 2 diabetes will likely continue to rise.

These individuals also represent a particularly high-risk group with poor long-term outcomes (including greater multimorbidity, risk of complications and years of life lost $[1,5,7,8,11])$. Distinct management strategies or approaches may therefore be optimal for this group. For example, they may benefit from earlier, more aggressive intervention to halt the early development of severe complications, whilst novel approaches to long-term management and ongoing clinical consultation may also be effective. Sex-specific strategies may also be warranted. However, trials specifically in individuals with type 2 diabetes aged 18-39 years are required to test these hypotheses. In this regard, the inclusion of 'interventions in the under 40s with type 2 diabetes' in the 2018 UK NHS Research Needs Assessment and accordant research recommendations from the National Institute for Health and Care Excellence are encouraging [15, 16]. Data from studies addressing these calls are eagerly anticipated.

Acknowledgements The authors thank C. Franklin (Diabetes Research Centre, University of Leicester, Leicester, UK) for his assistance with preparation of the figures contained in this manuscript and accompanying ESM. The views expressed are those of the authors and not necessarily those of the National Health Service, the National Institute for Health Research (NIHR) or the Department of Health.

Data availability The dataset generated during this study is available from the corresponding author on reasonable request.

Funding This research was supported by the NIHR Leicester Biomedical Research Centre, the NIHR Applied Research Collaboration East Midlands and the NIHR Programme Grants for Applied Research funding stream.

Duality of interest JAS has received a grant in support of an investigatorinitiated trial from AstraZeneca. FZ has acted as a speaker for Napp Pharmaceuticals. DRW has received honoraria as a speaker for AstraZeneca, Sanofi-Aventis and Lilly and has received research funding support from Novo Nordisk. VRA has served as a consultant for Adocia, AstraZeneca, BD, Novo Nordisk, Sanofi and Zafgen, with clinical trials/ research support to employer institution from AstraZeneca/BMS, Boehringer Ingelheim, Calibra, Eisai, Fractyl, Janssen, Novo Nordisk, Sanofi and Theracos. Her spouse is an employee of Merck Research Laboratories. KK has received honoraria from Abbot, AstraZeneca, Berlin-Chemie AG/Menarini Group, Boehringer Ingelheim, Eli Lilly, Janssen, MSD, Novartis, Novo Nordisk, Roche and Sanofi, and research support from AstraZeneca, Boehringer Ingelheim, Eli Lilly, Janssen, MSD, Novartis, Novo Nordisk, Roche and Sanofi. MJD has acted as a consultant, advisory board member and speaker for Novo Nordisk, Sanofi-Aventis, Lilly, MSD, Boehringer Ingelheim, AstraZeneca and Janssen, an advisory board member for Servier and a speaker for Mitsubishi Tanabe Pharma Corporation and Takeda Pharmaceuticals International Inc. She has received grants in support of investigator and investigator-initiated trials from Novo Nordisk, Sanofi-Aventis, Lilly, Boehringer Ingelheim, Janssen and AstraZeneca. EMB, FT and EWG report no conflicts of interest.

Contribution statement JAS, EMB, FZ and MJD contributed to the conception and design of the work. JAS, EMB and FT performed the search and data extraction. JAS performed the data analysis. JAS and MJD interpreted the analyses and drafted the manuscript. EMB, FZ, FT, DRW, VRA, EWG and KK reviewed the manuscript, providing substantial academic and/or clinical input and interpretation of findings. All authors approved the final manuscript. JAS is the guarantor of the work and as such had full access to all the data and takes responsibility for the integrity of the data and the accuracy of the data analysis. 
Open Access This article is licensed under a Creative Commons Attribution 4.0 International License, which permits use, sharing, adaptation, distribution and reproduction in any medium or format, as long as you give appropriate credit to the original author(s) and the source, provide a link to the Creative Commons licence, and indicate if changes were made. The images or other third party material in this article are included in the article's Creative Commons licence, unless indicated otherwise in a credit line to the material. If material is not included in the article's Creative Commons licence and your intended use is not permitted by statutory regulation or exceeds the permitted use, you will need to obtain permission directly from the copyright holder. To view a copy of this licence, visit http://creativecommons.org/licenses/by/4.0/.

\section{References}

1. Lascar N, Brown J, Pattison H, Barnett AH, Bailey CJ, Bellary S (2018) Type 2 diabetes in adolescents and young adults. Lancet Diabetes Endocrinol 6(1):69-80. https://doi.org/10.1016/S22138587(17)30186-9

2. Yeung RO, Zhang Y, Luk A et al (2014) Metabolic profiles and treatment gaps in young-onset type 2 diabetes in Asia (the JADE programme): a cross-sectional study of a prospective cohort. Lancet Diabetes Endocrinol 2(12):935-943. https://doi.org/10.1016/ S2213-8587(14)70137-8

3. Centres for Disease Control and Prevention (2020) National diabetes statistics report 2020. Estimates of diabetes and its burden in the United States. Available from www.cdc.gov/diabetes/pdfs/data/ statistics/national-diabetes-statistics-report.pdf. Accessed 20 Jul 2019

4. Nanayakkara N, Pease AJ, Ranasinha S et al (2018) Younger people with type 2 diabetes have poorer self-care practices compared with older people: results from the Australian National Diabetes Audit. Diabet Med 35(8):1087-1095. https://doi.org/10. 1111/dme. 13660

5. Chan JCN, Lau ESH, Luk AOY et al (2014) Premature mortality and comorbidities in young-onset diabetes: a 7-year prospective analysis. Am J Med 127(7):616-624. https://doi.org/10.1016/j. amjmed.2014.03.018

6. Lascar N, Altaf Q-A, Raymond NT et al (2019) Phenotypic characteristics and risk factors in a multi-ethnic cohort of young adults with type 2 diabetes. Curr Med Res Opin 35(11):1893-1900. https://doi.org/10.1080/03007995.2019.1638239
7. Song SH, Gray TA (2011) Early-onset type 2 diabetes: high risk for premature diabetic retinopathy. Diabetes Res Clin Pract 94(2):207211. https://doi.org/10.1016/j.diabres.2011.07.030

8. Sattar N, Rawshani A, Franzén S et al (2019) Age at diagnosis of type 2 diabetes mellitus and associations with cardiovascular and mortality risks findings from the Swedish National Diabetes Registry. Circulation 139(11):2228-2237. https://doi.org/10.1161/ CIRCULATIONAHA.118.037885

9. Browne JL, Scibilia R, Speight J (2013) The needs, concerns, and characteristics of younger Australian adults with type 2 diabetes. Diabet Med 30(5):620-626. https://doi.org/10.1111/dme.12078

10. Browne JL, Nefs G, Pouwer F, Speight J (2015) Depression, anxiety and self-care behaviours of young adults with type 2 diabetes: results from the International Diabetes Management and Impact for Long-term Empowerment and Success (MILES) Study. Diabet Med 32(1):133-140. https://doi.org/10.1111/dme.12566

11. Gregg EW, Cheng YJ, Srinivasan M et al (2018) Trends in causespecific mortality among adults with and without diagnosed diabetes in the USA: an epidemiological analysis of linked national survey and vital statistics data. Lancet 391(10138):2430-2440. https://doi.org/10.1016/S0140-6736(18)30314-3

12. Ke C, Shah B, Luk A, Di Ruggiero E, Chan J (2019) Cardiovascular outcomes trials in type 2 diabetes: time to include young adults. Diabetes Obes Metab 22(1):3-5. https://doi.org/10. 1111/dom. 13874

13. Davies MJ, D'Alessio DA, Fradkin J et al (2018) Management of hyperglycaemia in type 2 diabetes, 2018. A consensus report by the American Diabetes Association (ADA) and the European Association for the Study of Diabetes (EASD). Diabetologia 61(12):2461-2498. https://doi.org/10.1007/s00125-018-4729-5

14. Gemmill JAL, Brown RJ, Nandagopal R, Rodriguez LM, Rother KI (2011) Clinical trials in youth with type 2 diabetes. Pediatr Diabetes 12(1):50-57. https://doi.org/10.1111/j.1399-5448.2010. 00657.x

15. NHS England (2018) NHS England's Research Needs Assessment 2018. Available from www.england.nhs.uk/publication/nhsenglands-research-needs-assessment-2018. Accessed 20 Jul 2019

16. National Institute for Health and Care Excellence (2019) Research recommendations. Available from www.nice.org.uk/about/whatwe-do/science-policy-research/research-recommendations. Accessed 20 Jul 2019

Publisher's note Springer Nature remains neutral with regard to jurisdictional claims in published maps and institutional affiliations. 